\title{
Mental Disorders and Emotional Competence Among Chinese Adolescents Before and During COVID-19 Pandemic: A Longitudinal Mediation Model
}

OPEN ACCESS

Edited by:

Chang Liu,

University of Texas at Austin,

United States

Reviewed by:

Konstantinos Kotsis,

University of loannina, Greece

Emmanouil Tsalamanios,

General Hospital Asklepieio

Voulas, Greece

*Correspondence:

Li Zhao

zhaoli@scu.edu.cn

Peng Jia

jiapengff@hotmail.com

Specialty section:

This article was submitted to

Public Mental Health,

a section of the journal

Frontiers in Public Health

Received: 30 August 2021 Accepted: 18 November 2021 Published: 10 December 2021

Citation:

Shi W, Yuan GF, Hall BJ, Liu X, Su Y,

Zhao $L$ and Jia $P$ (2021) Mental Disorders and Emotional Competence Among Chinese Adolescents Before and During COVID-19 Pandemic: A Longitudinal Mediation Model.

Front. Public Health 9:767004. doi: 10.3389/fpubh.2021.767004

\begin{abstract}
Wei Shi ${ }^{1}$, Guangzhe Frank Yuan ${ }^{2}$, Brian J. Hall ${ }^{3,4}$, Xiaoli Liu ${ }^{5}, \mathrm{Ya} \mathrm{Su}^{6}, \mathrm{Li}_{\mathrm{ZhaO}}{ }^{7,8 *}$ and Peng Jia ${ }^{1,9,10 *}$

${ }^{1}$ Institute for Disaster Management and Reconstruction (IDMR), Sichuan University, Chengdu, China, ${ }^{2}$ Department of Psychology, Faculty of Social Sciences, University of Macau, Macau SAR, China, ${ }^{3}$ Global and Community Mental Health Research Group, New York University, Shanghai, China, ${ }^{4}$ Department of Health, Behavior and Society, Johns Hopkins Bloomberg School of Public Health, Baltimore, MD, United States, ${ }^{5}$ Office of Humanities and Social Sciences Administration, Sichuan University, Chengdu, China, ${ }^{6}$ Department of Sociology, Dickinson College, Carlisle, PA, United States, ${ }^{7}$ Department of Health Policy and Management, West China School of Public Health and West China Fourth Hospital, Sichuan University, Chengdu, China, ${ }^{8} \mathrm{Health}$ Emergency Management Research Center. China-PUMC C.C. Chen Institute of Health, Sichuan University, Chengdu, China, ${ }^{9}$ School of Resources and Environmental Science, Wuhan University, Wuhan, China,

${ }^{10}$ International Institute of Spatial Lifecourse Epidemiology (ISLE), Wuhan University, Wuhan, China
\end{abstract}

Background: The outbreak of the COVID-19 pandemic has had a profound influence on the mental health and well-being of individuals across the globe. Emotional competence, defined as one's ability to recognize, understand, and manage their emotions, has been found linked with mental health problems (e.g., depression and anxiety) in previous studies. However, there is limited knowledge about the direction of the association between these factors among populations exposed to COVID-19. This study examined the possible mediation relationships between depression, anxiety, emotional competence, and COVID-19 exposure among Chinese adolescents.

Methods: Responses from 7,958 Chinese adolescents who had previously taken part in a two-wave study before (December 23, 2019-January 13, 2020) and during COVID-19 (June 16, 2020-July 8, 2020) were analyzed (51.67\% males, mean age $=11.74$, $\mathrm{SD}=2.15$ ). Structural equation modeling with three covariates (i.e., age, gender, and ethnicity) was used to test the longitudinal mediation relationships between COVID-19 exposure and depression, anxiety via emotional competence.

Results: Results indicated that the prevalence of depression (38.67 to 36.74\%) and anxiety (13.02 to $12.77 \%$ ) decreased from Time 1 to Time 2. The T2 emotional competence significantly mediated the relationship between T2 COVID-19 exposure and T2 anxiety (indirect effect $[95 \% \mathrm{Cl}]=0.011$ [0.004-0.019], $p<0.05$ ). T2 emotional competence also significantly mediated the relationship between T2 COVID-19 exposure and T2 depression (indirect effect [95\% Cl] $=0.013$ [0.005-0.022], $p<0.05$ ). The results indicated that T2 emotional competence had a significant and negative influence on T2 anxiety $(\beta=-0.266$, SE $=0.005, p<0.001)$, and T2 depression $(\beta=-0.326$, $\mathrm{SE}=0.029, p<0.001)$. 
Conclusions: This longitudinal research study demonstrated the crucial role of emotional competence in influencing the severity of long-term mental health problems, and suggested that emotional competence interventions can be conducted to improve mental well-being among Chinese adolescents exposed to COVID-19.

Keywords: anxiety, depression, emotional competence, longitudinal mediation model, COVID-19 exposure

\section{INTRODUCTION}

Adolescence is a vulnerable dangerous period during which mental disorders (e.g., depression and anxiety) can easily present themselves, increasing the risk of life-long mental illnesses (1). According to a report by the World Health Organization (2), 10 to $20 \%$ of adolescents suffer from mental problems worldwide, and most are underdiagnosed and undertreated (3). Being the fourth and sixth major causes of mental illness and disability among adolescents, depression and anxiety, respectively, are considered to be highly prevalent (2). For example, a meta-analysis of 17,894 subjects found that the prevalence of depression and anxiety was 17.96 and $13.99 \%$, respectively, in Chinese adolescents (4). In the USA, anxiety is the most common mental disorder, with a prevalence of $31.9 \%$ among 10,123 adolescents aged 1318 years (5). Additionally, an Australian research with a sample of 1,299 adolescents identified that the prevalence of depression and anxiety was 14.2 and $13.2 \%$, respectively (6). Most previous research has applied a cross-sectional design to explore mental health and its correlates among adolescents, but this approach lacks the long-term tracking of mental health status among the target population $(7,8)$. Therefore, it is crucial to explore the potential mechanisms associated with long-term mental disorders and related factors among adolescents.

Many prior studies have shown that public emergencies have a profound effect on mental health (9-12). In the UK, a national longitudinal cohort study with a sample of 53,351 participants indicated that the prevalence of mental distress increased from $18.9 \%$ before COVID-19 to $27.3 \%$ during COVID-19 pandemic (13). In Switzerland, a study found that levels of anxiety and depressive symptoms significantly worsened among undergraduate students after COVID-19, compared with those before COVID-19 (14). However, research in the Netherlands showed that mental health variables remained approximately the same before and during COVID-19 among 141 adolescent students (15). Another longitudinal study among 203 Chinese students reported a significant decrease in anxiety and depression during COVID-19 lockdown (16). Furthermore, an American study, which included 322 young adolescents, found that for participants with a good mental health (i.e., fewer emotional problems before COVID-19), psychological symptoms significantly decreased during COVID-19 pandemic (17). Existing literature on mental health developing trend and related variables remained debatable before and during COVID19 pandemic. Hence, it is necessary to further track long-term mental health status, and explore the influence of exposure to public emergencies on the mental health of Chinese adolescents, before and during COVID-19.

Emotional competence (EC) is broadly defined as an individual's ability to recognize, understand, and manage their emotions (18). In recent years, more research has underscored the vital role of EC in psychopathology, such as depression and anxiety (18-21). For example, previous meta-analyses have shown that individuals with higher EC are associated with lower levels of psychological distress, including depression and anxiety $(20,22-24)$. A previous study highlighted that a higher level of EC was associated with greater well-being and a lower risk of developing mental disorders (25). Moreover, an 18-h EC intervention experiment showed that improvement in EC promoted positive changes in psychological well-being (26). Most previous studies on EC applied a cross-sectional design and there has been a lack of studies examining the ECmediated relationship between depression and anxiety based on longitudinal data. Thus, it is necessary to further explore the directional association between EC, depression, and anxiety, using a longitudinal mediation model.

Several previous theories and models have shown the potential influence mechanism of EC for mental disorders. First, according to the transdiagnostic emotion dysregulation model of mood and anxiety disorders, a triggering event could connect with the present diathesis and result in a negative or positive influence. The final psychological impact could depend on an individual's emotional style and recognition (27). Mood disorders result from emotional dysregulation of negative influences and are associated with an absence of positive affect (28). Second, the transdiagnostic models of psychopathology explain the mechanisms by which transdiagnostic risk factors result in multiple mental disorders (29). The model suggested that biological factors giving rise to potentially maladaptive emotional and cognitive trends could directly result in psychological symptoms, such as depression and anxiety (30). Finally, the ABC theory of emotion proposed by Ellis (31) holds that emotions can be directly determined by the individual evaluation and cognition processes of the triggering event. Emotional evaluation and recognition are greatly influenced by EC, which can lead to various emotional reactions and changes (20). Hence, based on the research gaps and theoretical foundation, this study aimed to explore the possible mediation relationships between depression, anxiety, emotional competence, and COVID-19 exposure, and understand the long-term developmental trend of mental health status among Chinese adolescents through a longitudinal design study, before and during COVID-19. This longitudinal analysis involved six major variables including T1 depression, T2 depression, T1 anxiety T2 anxiety, T2 emotional competence, 
and T2 COVID-19 exposure. As previous research suggested that people with different mental disorders (e.g., depression and anxiety) before COVID-19 could display dissimilar developing trends in psychological status during COVID-19 (15). It is imperative to examine the mental health symptoms (i.e., T1 depression and T1 anxiety) before COVID-19 and accurately detect changes in mental health via a longitudinal study during the pandemic.

\section{MATERIALS AND METHODS}

\section{Dataset}

A face-to-face interview questionnaire was used to collect data from students in two middle schools and three high schools. Data were collected from two-wave studies named Chengdu Positive Child Development (CPCD) survey (8). Time 1 (T1) data were collected between December 23, 2019, and January 13, 2020, before the outbreak of COVID-19 in China. Time 2 (T2) data were collected between June 16, 2020, and July 8, 2020, when the epidemic was under control in China and schools were re-opened. All participants were informed of the research purpose, privacy measures, and data retained in the signed consent form. This study was approved by the Research Ethics Committee of the University. Questionnaires were distributed to 10,370 participants. A total of 8,749 valid questionnaires were returned in the $\mathrm{T} 1$ study $\left(51.62 \%\right.$ males, $\mathrm{M}_{\mathrm{age}}=12.02, \mathrm{SD}=2.30$, response rate $=84.37 \%)$. A total of 7,958 participants completed the second-wave T2 $\left(51.67 \%\right.$ males, $\mathrm{M}_{\mathrm{age}}=11.74, \mathrm{SD}=2.15$, response rate $=76.74 \%$ ). A total of 791 participants were lost to follow-up at T2. This study only included a sample of 7,958 participants who completed the two-wave study.

\section{Measures}

\section{Depression}

The past-week symptoms of depression were measured using the Chinese version of the Center for Epidemiologic Studies Depression Scale (CES-D) in both wave studies (32). The depression scale consists of 20 items, each rated on a four-point scale from 0 (not at all) to 3 (a lot). An example item is, "I felt lonely, and I don't have a lot of friends." Higher scores indicated a higher severity of depressive symptoms and total scores over 15 can indicate significant levels of depressive symptoms. The Chinese version of the CES-D has good validity and reliability in Chinese samples $(32,33)$. In this study, the scale showed good internal consistency (Cronbach's alphas were $>0.87$ in both wave data).

\section{Anxiety}

The last 3-month symptoms of anxiety were assessed using a subscale (Generalized Anxiety Disorder) of the Chinese Screen for Child Anxiety Related Emotional Disorders (SCARED) in each wave (34). The subscale contains nine items in total. Participants rated each item on a three-point scale from 0 (never) to 2 (often). A sample item is, "I worry about whether other people like me." Higher scores indicated a greater level of anxiety. Total scores over 9 can indicate significant levels of anxiety symptoms. The Chinese version of the SCARED has been shown to have good validity and reliability in previous studies $(34,35)$. In the current study, the internal consistency for this measure was good (Cronbach's alphas were $>0.86$ in both wave data).

\section{Emotional Competence}

Emotional competence was measured using a subscale of the Chinese Positive Youth Development Scale (CPYDS) in the second wave of the study (36). The CPYDS included 90 items, in which a six-item subscale was used to examine emotional competence. Each item is rated on a six-point scale ranging from 1 (strongly disagree) to 6 (strongly agree). An example item is "When I am unhappy, I can appropriately show my emotions." The reliability and validity of the subscale were established in previous studies $(36,37)$. In this study, the subscale showed good internal consistency (Cronbach's alpha was 0.86 ).

\section{COVID-19 Exposure}

Based on the context of the participant population and previous studies $(38,39)$, nine items were developed to test the COVID-19 exposure in the second wave of the study. Four items with a four-point scale $(1=$ not at all; 4 $=$ extremely severe/dangerous/possible/ability) examined the perceived severity, danger, infection risk, and prevention ability for COVID-19. One dichotomous question tested whether the participant's family had been infected with COVID-19 $(1=$ no; $2=$ yes). Additionally, four questions with a four-point scale ( 1 $=$ not at all; $4=$ extreme influence) assessed the effects of diet, study, social life, and recreational activities. The total score was the sum of all items, with a higher score indicating a higher level of COVID-19 exposure.

\section{Statistical Analysis}

There were four aspects in the data analysis using SPSS Version 24.0 (40) and AMOS Version 23.0 (41). First, descriptive analyses and correlations were conducted for all the variables. Second, the independent samples $t$-test was used to examine the differences between 7,958 participants who completed the two-wave study and 791 respondents who were lost to followup in the T2 study among all variables. Third, a paired-samples $t$-test was used to test the differences in levels of depression and anxiety between the first and second waves of the study. Finally, structural equation modeling was conducted to test the longitudinal mediation relationships between depression, anxiety, and emotional competence. Six potential mediation pathways were tested. Four variables-depression, anxiety, emotional competence, and COVID-19 exposure were modeled as latent variables in the current study. In addition, three covariates, including age, gender, and ethnicity, were added to examine the mediation model since they could be correlated with depression, anxiety, and emotional competence. To test the hypothesized mediation effect for statistical significance in AMOS, bootstrapping was used via 5,000 bootstrapped replications. Parameters were examined through a maximum likelihood estimation. According to prior research (42), $\chi^{2}$ statistics are usually applied for testing model fit, but they could be largely influenced by the sample size. Thus, other model fit indices were recommended to further assess the goodness fit of 
the model via several indexes (43), including $\chi^{2} / \mathrm{df}$, root mean square error of approximation (RMSEA), standardized root mean square residual (SRMR), Tucker-Lewis Index (TLI), and comparative fit index (CFI). According to the recommendation of the acceptable model index, the ratio of $\chi^{2}$ to the degree of freedom should be $<5.0$, TLI and CFI should be higher than 0.95, and SRMR and RMSEA should be smaller than 0.05 (42).

\section{RESULTS}

\section{Descriptive Findings}

The descriptive statistics, including the means, standard deviations, and intercorrelations among study variables, are displayed in Tables 1, 2. There were significant correlations between depression, anxiety, emotional competence, and COVID-19 exposure across the study waves. All scale scores showed a significantly moderate or high intercorrelation, excluding the COVID-19 exposure at T2, which was marginally correlated with emotional competence at T2 $(r=-0.089$,

TABLE 1 | Demographics $(n=7,958)$.

\begin{tabular}{lc}
\hline Variable & (N, \%) \\
\hline $\begin{array}{l}\text { Age (7-17) } \\
\text { Gender }\end{array}$ & $\mathrm{M}=11.74, \mathrm{SD}=2.15$ \\
Male & $4,112(51.67 \%)$ \\
Female & $3,846(48.33 \%)$ \\
Ethnicity & $7,893(99.2 \%)$ \\
Han Chinese & $65(0.8 \%)$ \\
Other Ethnicities & $\mathrm{M}=21.00, \mathrm{SD}=4.05$ \\
T2 coviD-19 Exposure & $\mathrm{M}=28.38, \mathrm{SD}=6.67$ \\
T2 Emotional Competence & \\
Depression & $\mathrm{M}=14.40, \mathrm{SD}=10.16, \mathrm{~N}_{\text {Yes }}=3,078(38.68 \%)$ \\
T1 & $\mathrm{M}=14.36, \mathrm{SD}=10.62 \mathrm{~N}_{\text {Yes }}=2,924(36.74 \%)$ \\
T2 & \\
Anxiety* & $\mathrm{M}=3.71, \mathrm{SD}=3.96 \mathrm{~N}_{\text {Yes }}=1,036(13.02 \%)$ \\
T1 & $\mathrm{M}=3.33, \mathrm{SD}=4.07 \mathrm{~N}_{\text {Yes }}=1,016(12.77 \%)$ \\
T2 &
\end{tabular}

M, Mean; SD, Standard Deviation; $N_{\text {yes }}$, number of participants with anxiety or depression; ${ }^{*} p<001$ (Paired-sample t-test); T1, Time 1; T2, Time 2. $p<0.001)$ after adjusting for age, gender, and ethnicity. The prevalence of depression decreased from $38.68 \%(n=3,078)$ in T1 to $36.74 \%(n=2,924)$ in T2. Similarly, the prevalence of anxiety significantly decreased from $13.02 \%(n=1,036)$ in T1 to $12.77 \%(n=1,016)$ in T2 $(p<0.001)$. Additionally, no significant differences, except for age and anxiety, were found between the study population $(n=7,958)$ and the participants lost to follow-up, based on all variables $\left(p_{s}>0.001\right)$.

\section{Longitudinal Mediation Model}

The results showed the mediation model without three covariates (i.e., age, gender, and ethnicity) and had excellent model fit ( $\chi^{2} / \mathrm{df}$ $=4.029, \mathrm{CFI}=0.984, \mathrm{TLI}=0.982, \mathrm{SRMR}=0.037$, RMSEA $[90 \%$ $\mathrm{CI}]=0.020[0.019-0.020])$. The model fit remained excellent after including covariates, age, gender, and ethnicity status $\left(\chi^{2} / \mathrm{df}\right.$ $=4.974, \mathrm{CFI}=0.976, \mathrm{TLI}=0.973, \mathrm{SRMR}=0.038, \mathrm{RMSEA}[90 \%$ $\mathrm{CI}]=0.022[0.022-0.023]$ ) (see Figure 1). Two relationships were significantly mediated by T2 emotional competence. First, T2 emotional competence significantly mediated the relationship between T2 COVID-19 exposure and T2 anxiety (indirect effect $[95 \% \mathrm{CI}]=0.011[0.004-0.019], p<0.05)$. Second, T2 emotional competence significantly mediated the relationship between $\mathrm{T} 2$ COVID-19 exposure and T2 depression (indirect effect [95\% CI] $=0.013[0.005-0.022], p<0.05)$.

The longitudinal mediation model estimated the association between COVID-19 exposure, emotional competence, anxiety, and depression across the two waves, in which age, gender, and ethnicity were covariates. The path coefficients of the model are displayed in Figure 1, which indicates several significant direct paths, including autoregressive paths from T1 anxiety to T2 anxiety $(\beta=0.461, \mathrm{SE}=0.016, p<0.001)$ and $\mathrm{T} 1$ depression to T2 depression $(\beta=0.442, \mathrm{SE}=0.014, p<0.001)$, and direct effects from T2 COVID-19 exposure to T2 anxiety ( $\beta=0.114$, SE $=0.006, p<0.001)$, T2 COVID-19 exposure to T2 depression $(\beta=0.107, \mathrm{SE}=0.031, p<0.001), \mathrm{T} 2 \mathrm{COVID}-19$ exposure to $\mathrm{T} 2$ emotional competence $(\beta=-0.041, \mathrm{SE}=0.015, p<0.001)$, $\mathrm{T} 2$ emotional competence to $\mathrm{T} 2$ anxiety $(\beta=-0.266, \mathrm{SE}=$ $0.005, p<0.001$ ), and T2 emotional competence to T2 depression $(\beta=-0.326, \mathrm{SE}=0.029, p<0.001)$.

Age was significantly associated with $\mathrm{T} 2$ anxiety $(\beta=-0.100$, $\mathrm{SE}=0.002, p<0.001)$, T2 emotional competence $(\beta=-0.061$, $\mathrm{SE}=0.005, p<0.001)$, and $\mathrm{T} 2$ depression $(\beta=0.068, \mathrm{SE}=$ $0.011, p<0.001)$. Gender was significantly associated with T2

TABLE 2 | Means, standard deviations, and correlations among study variables $(n=7,958)$.

\begin{tabular}{|c|c|c|c|c|c|c|c|c|}
\hline$\#$ & Variables & $M \pm S D$ & 1 & 2 & 3 & 4 & 5 & 6 \\
\hline 1 & T1 Depression & $14.40 \pm 10.15$ & - & $0.613^{*}$ & $0.530^{*}$ & $0.412^{*}$ & $-0.350^{*}$ & $0.135^{\star}$ \\
\hline 2 & T1 Anxiety & $3.71 \pm 3.96$ & $0.607^{\star}$ & - & $0.424^{*}$ & $0.504^{\star}$ & $-0.298^{\star}$ & $0.138^{*}$ \\
\hline 4 & T2 Anxiety & $3.33 \pm 4.07$ & $0.405^{\star}$ & $0.518^{*}$ & $0.635^{\star}$ & - & $-0.361^{\star}$ & $0.187^{*}$ \\
\hline 5 & T2 EC & $28.38 \pm 6.67$ & $-0.348^{\star}$ & $-0.303^{*}$ & $-0.451^{*}$ & $-0.366^{\star}$ & - & $-0.089^{*}$ \\
\hline
\end{tabular}

Left/bottom triangle is the Pearson's correlations of all study variables, right/top triangle is the partial correlation of all the variables adjusted by age, gender, and ethnicity; M, Mean; SD, Standard Deviation; EC, Emotional Competence; COVID-19, COVID-19 Exposure; ${ }^{*} p<0.001$. 


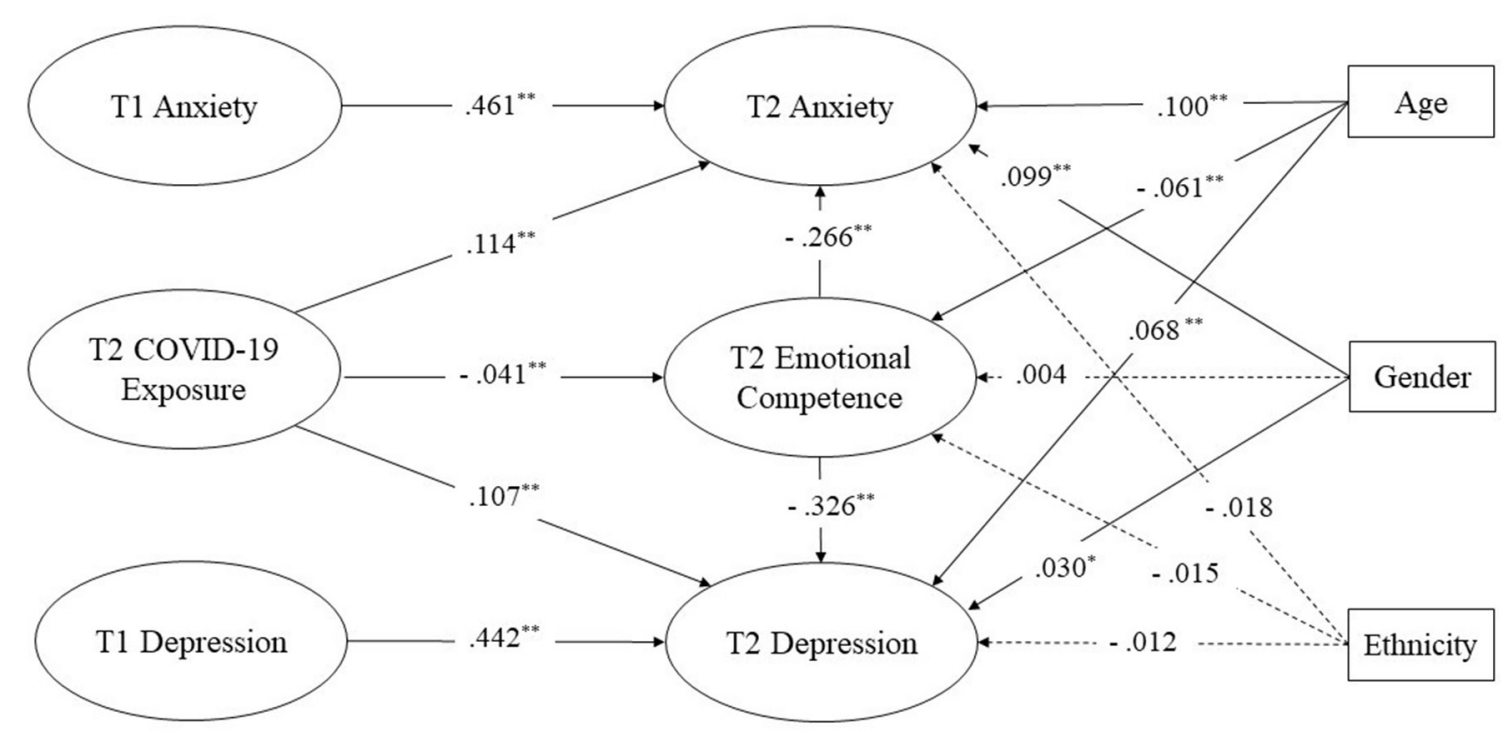

FIGURE 1 | Longitudinal mediation model with standardized path coefficients. ${ }^{*} p<0.05,{ }^{* *} p<0.001$; For simplicity, factor loadings of all items are not displayed in this figure. Dotted lines displayed the non-significant paths; Solid lines displayed the significant paths.

anxiety $(\beta=0.099, \mathrm{SE}=0.009, p<0.001)$ and T2 Depression $(\beta$ $=0.030, \mathrm{SE}=0.047, p<0.05)$. However, four non-significant paths were found in this study. Ethnicity did not significantly associate with $\mathrm{T} 2$ anxiety $(\beta=-0.018, \mathrm{SE}=0.047, p=$ $0.080)$, T2 emotional competence $(\beta=-0.015, \mathrm{SE}=0.125, p$ $=0.169)$, and $\mathrm{T} 2$ depression $(\beta=-0.012, \mathrm{SE}=0.263, p=$ $0.218)$, respectively. Gender was not a significant predictor of T2 emotional competence $(\beta=0.004, \mathrm{SE}=0.023, p=0.700)$.

\section{DISCUSSION}

This longitudinal study explored the associations between depression, anxiety, emotional competence, and COVID-19 exposure among Chinese adolescents before and during COVID19. To our knowledge, this is the first longitudinal study to highlight the vital mediating role of emotional competence as a possible mechanism underlying the association between COVID19 exposure and long-term mental disorders of depression and anxiety. Additionally, the results further evidenced the negative influence of COVID-19 exposure on long-term mental health among Chinese adolescents. The results may provide a better understanding of psychological disorder development trends related to public health emergencies, highlighting the role of emotional competence as a mediator. This could benefit the development of more emotional competence interventions to improve future mental well-being among adolescents exposed to public health emergencies.

Based on the hypothesized mediation model, one of the major findings was that 6-month follow-up levels of emotional competence played a vital mediating role in the association between 6-month follow-up depression and COVID-19 exposure after controlling for gender, age, and ethnicity. Most prior studies on mental health in the COVID-19 preferred to explore its correlation between outcome variables without including potential mediators of psychological disorders $(44,45)$. The results from the current study suggested that emotional ability related variables, particularly the ones examining the levels of emotional skills or capabilities, could be included when exploring long-term psychological disorders in the context of the COVID-19 pandemic. It is imperative for researchers to consider variables related to emotional ability in studies on adolescents. This is because when young people are better able to manage their emotions, they are more likely to seek mental support from professionals and are more likely to recover from mental disorders (46). Other vital elements of emotional ability include the knowledge to identify mental health problems, the capacity to seek help, and the capability to recognize mental health issues (26). These aspects could be included in future studies to examine their effect on relieving long-term mental disorders among adolescents exposed to public emergencies. Moreover, this current study displayed a significantly negative association between depression and emotional competence at the 6-month follow-up, which suggested that adolescents with a higher emotional competence are likely to have lower levels of depression. This is consistent with previous research which showed that people with a better ability to recognize mental disorders are less likely to suffer from mental disorders when exposed to the COVID-19 pandemic (47). The results further emphasized the importance of improving emotional competence, such as improving Chinese adolescents' ability to recognize and modify their negative emotions.

This study found at the 6-month follow-up, that levels of emotional competence significantly mediated the association between anxiety and COVID-19 exposure. These results are consistent with the study hypotheses and supported 
by the transdiagnostic emotion dysregulation model which states that increasing emotional competence influences the association between early indication of psychological distress (e.g., depression and anxiety) and relieving psychological distress later $(27,48-50)$. This pattern also matches the transdiagnostic models of psychopathology, which claim that better emotional trends and abilities help diminish mental disorders $(29,30)$. For individuals with early mental health problems, future well-being can be safeguarded by improving their emotional competence (22). Additionally, there was a significant negative association at the 6-month follow-up between emotional competence and COVID-19 exposure. A meta-analysis with 31 cross-sectional studies reported a slightly higher overall prevalence of anxiety (24\%) than depression (22\%) among adolescents exposed to COVID-19 pandemic (12). These results suggested that promoting emotional competence could be an effective way to help adolescents with anxiety symptoms when suffering from public emergencies.

Unexpectedly, this study found that the prevalence of depression and anxiety decreased among Chinese adolescents after exposure to the COVID-19 pandemic. This result is different from the findings of most previous studies that reported the increasing of psychological disorders during COVID-19 pandemic $(13,14)$. One possible explanation is that adolescents with severe mental health problems were able to seek more emotional support from families or friends, increasing their emotional ability, which resulted in a decrease in the occurrence or severity of future mental health problems (51). Moreover, previous research suggested that people could receive more emotional support from society under triggering events, such as COVID-19 pandemic, than during routine times (52). When COVID-19 pandemic occurred, Chinese society provided considerable mental health support for exposed individuals, which could promote emotional competence and relieve mental disorders (53). This study also demonstrated an increase in emotional competence among Chinese adolescents after exposure to COVID-19 pandemic, which possibly resulted from the improvement in social and mental health support resources during COVID-19 pandemic in China (53). Prior research has shown that long-term mental health problems can be decreased when more emotional recognition is received (23).

This result indicating decreased mental disorders aligned with the results of smaller research among Chinese and American populations $(16,17)$. There are some possible explanations for this result. For example, the level of depression and anxiety decreased in Chinese adolescents because of decreased academic pressure (54). A major reason for depression and anxiety among Chinese adolescents is their interpersonal relationships (55). Many Chinese adolescents had to study at home because of COVID-19 pandemic, and they could avoid academic stress and difficult interpersonal relationships at school. Therefore, the prevalence of mental disorders could be reduced during the COVID-19 period compared with the pre-COVID-19 period (16). Moreover, a previous study suggested that COVID-19 stayat-home regulations could provide a protective effect and context for the mental health of youth (17). Thus, there was a decreasing trend of depression and anxiety among Chinese adolescents because they could have gained more mental health support from their families during COVID-19 than pre-COVID-19 pandemic, because they were likely to have had more time with their family members at home (56).

The results suggested that ethnicity was not a significant predictor of depression, anxiety, and emotional competence in the 6-month follow-up, which was inconsistent with previous research which suggested that being an ethnicity could influence mental health and emotional competence $(57,58)$. One possible explanation for these results was that the differences between the other minorities and the Hans (viz., The ethic Han Chinese accounts for more than $90 \%$ of the total population in China) are narrowing because of the educational improvement, economic development, and acculturation within the Chinese population (59), which suggests that ethnic differences did not influence the mental status and emotional competence education. Furthermore, the percentage of ethnic minority participants was low in this study, compared with the Han participants, which possibly resulted in underestimating the influence of minorities on outcome variables in the structural equation model (60). Further research is suggested to balance the percentage of the minorities and the Han participants when exploring related research topics.

Based on a large sample of Chinese adolescents, the current study applied well-validated measures to explore the associations between emotional competence, depression, anxiety, and COVID-19 exposure via a complete longitudinal mediation design. The results underscored the vital role of emotional competence in influencing long-term mental disorders, such as depression and anxiety, among Chinese adolescents exposed to COVID-19 pandemic. Despite these merits, this study has several limitations that warrant discussion. First, this study utilized face-to-face interview questionnaires to collect data, which could result in response bias because of the different qualities of the interviewer (61). Future studies should apply multiple approaches to collect data, such as mixed-method research. Second, this study was based on a two-wave study to explore the mediating effect of emotional competence on the association between COVID-19 exposure and mental disorders. There are other possible factors influencing long-term mental disorders among Chinese adolescents exposed to COVID-19 pandemic, such as social support (10), psychological help-seeking (62), social media usage (63), and resilience (64). Future research is suggested to explore more possible factors relieving longterm mental disorders among adolescents exposed to COVID19 pandemic. Finally, the mediation model could not prove the causal relationships between the variables without using the experimental method. A future study is recommended to explore the related intervention of emotional competence and its effect on long-term mental disorders among adolescents based on an experimental design (65).

\section{CONCLUSIONS}

This study emphasized emotional competence as an effective alleviative variable against long-term depression and anxiety 
among Chinese adolescents exposed to COVID-19 pandemic, the negative association between psychological distress (i.e., depression and anxiety) and emotional competence, and the long-term influence of improving emotional competence on mental health when exposed to COVID-19 pandemic. Additionally, the study found a decreased trend of mental disorders, including depression and anxiety among Chinese adolescents before and during the COVID-19 pandemic, which suggests that more variables, such as family support (51), receiving social information (63), and quarantine policies (10), need to be explored for releasing the mental burden among the population exposed to COVID-19 pandemic. Concerning psychological practice, this study suggested that more government- or school-based online lectures, training, or guidebooks about improving emotional competence should be provided for adolescents exposed to the COVID-19 pandemic, which could protect exposed individuals from exacerbating mental disorders. Moreover, intervention programs targeted at reinforcing emotional competence during or after COVID19 pandemic are necessary, which could be beneficial for strengthening emotional recognition and resilience and assisting in mental health and adaptability in future public health emergency events. For example, Kumschick and his colleagues (66) designed a literature-based intervention, named READING AND FEELING, to increase emotional competence. The results demonstrated the effectiveness of the READING AND FEELING program in improving emotional ability, based on emotional words, specific emotional literacy, and recognition of disguised moods among the youth. Furthermore, another intervention, Health Promoting Schools Up, was developed to effectively boost mental health by improving social and emotional competence among schoolchildren (67). Many researchers have recommended that various health support materials be provided for individuals exposed to COVID-19 pandemic to potentially relieve their long-term mental health disorders that develop due to this pandemic. These include online peersupport interventions (68), cyber-counseling (69), and digital mental health services (70), which could help to detect early

\section{REFERENCES}

1. Barrett P, Turner C. Prevention of anxiety symptoms in primary school children: preliminary results from a universal school-based trial. Br J Clin Psychol. (2001) 40:399-410. doi: 10.1348/014466501163887

2. World Health Organization. Adolescent Mental Health. (2020). Available online at: https://www.who.int/news-room/fact-sheets/detail/adolescentmental-health (accessed September 28, 2020).

3. Kessler RC, Angermeyer M, Anthony JC, De Graaf RON, Demyttenaere K, Gasquet I, et al. Lifetime prevalence and age-of-onset distributions of mental disorders in the World Health Organization's World Mental Health Survey initiative. World Psychiatry. (2007) 6:168-76.

4. Wang S, Sun Q, Zhai L, Bai Y, Wei W, Jia L. The prevalence of depression and anxiety symptoms among overweight/obese and non-overweight/nonobese children/adolescents in China: a systematic review and meta-analysis. Int J Environ Res Public Health. (2019) 16:340. doi: 10.3390/ijerph160 30340

5. Merikangas KR, He J-P, Burstein M, Swanson SA, Avenevoli S, Cui $\mathrm{L}$, et al. Lifetime prevalence of mental disorders in US adolescents: psychological problems and avoid mental symptom deterioration $(71-74)$.

\section{DATA AVAILABILITY STATEMENT}

The raw data supporting the conclusions of this article will be made available by the authors, without undue reservation.

\section{ETHICS STATEMENT}

The studies involving human participants were reviewed and approved by Research Ethics Committee of the University. Written informed consent to participate in this study was provided by the participants' legal guardian/next of kin.

\section{AUTHOR CONTRIBUTIONS}

PJ and LZ did study design and data collection. WS and GY analyzed data, drafted, and submitted this manuscript together. PJ, LZ, BH, YS, and XL revised the manuscript. All authors contributed to manuscript checking and approval the final manuscript.

\section{FUNDING}

This work was supported by the Fundamental Research Funds for the Central Universities (20827044B4020) and the International Institute of Spatial Lifecourse Epidemiology (ISLE).

\section{ACKNOWLEDGMENTS}

We sincerely appreciate all participants of this research, volunteers for the data collection, and administrative colleagues at all levels who helped us to finish this research project. More importantly, we are particularly grateful to all front-line workers around the world for bravely fighting this COVID-19 pandemic and protecting our lives. results from the National Comorbidity Survey Replication-Adolescent Supplement (NCS-A). J Am Acad Child Adolesc Psychiatry. (2010) 49:980-9. doi: 10.1016/j.jaac.2010.05.017

6. Boyd CP, Kostanski M, Gullone E, Ollendick TH, Shek DT. Prevalence of anxiety and depression in Australian adolescents: comparisons with worldwide data. J Genet Psychol. (2000) 161:479-92. doi: 10.1080/00221320009596726

7. Bitton MS, Laufer A. Mental health and coping in the shadow of the COVID-19 pandemic: the Israeli case. Front Public Health. (2020) 8:568016. doi: 10.3389/fpubh.2020.568016

8. Zhao L, Shek DTL, Zou K, Lei Y, Jia, P. Cohort profile: Chengdu Positive Child Development (CPCD) survey. Int J Epidemiol. (2021) 50:dyab237. doi: 10.1093/ije/dyab237

9. Meda N, Pardini S, Slongo I, Bodini L, Zordan MA, Rigobello P, et al. Students' mental health problems before, during, and after COVID-19 lockdown in Italy. J Psychiatr Res. (2021) 134:69-77. doi: 10.1016/j.jpsychires.2020.12.045

10. Shi W, Hall BJ. What can we do for people exposed to multiple traumatic events during the coronavirus pandemic? Asian J Psychiatry. (2020) 51:102065. doi: 10.1016/j.ajp.2020.102065 
11. Cindrich SL, Lansing JE, Brower, CS, McDowell CP, Herring MP, Meyer JD. Associations between change in outside time pre-and post-COVID-19 public health restrictions and mental health: brief research report. Front Public Health. (2021) 9:8. doi: 10.3389/fpubh.2021.619129

12. Zhang Y, Bao X, Yan J, Miao H, Guo C. Anxiety and depression in Chinese students during the COVID-19 pandemic: a meta-analysis. Front Public Health. (2021) 9:697642. doi: 10.3389/fpubh.2021.697642

13. Pierce M, Hope H, Ford T, Hatch S, Hotopf M, John A, et al. Mental health before and during the COVID-19 pandemic: a longitudinal probability sample survey of the UK population. Lancet Psychiatry. (2020) 7:883-92. doi: 10.1016/S2215-0366(20)30308-4

14. Elmer T, Mepham K, Stadtfeld C. Students under lockdown: comparisons of students' social networks and mental health before and during the COVID-19 crisis in Switzerland. PloS ONE. (2020) 15:e0236337. doi: 10.1371/journal.pone.0236337

15. Van Zyl LE, Rothmann S, Zondervan-Zwijnenburg M. A Longitudinal trajectories of study characteristics and mental health before and during the COVID-19 lockdown. Front Psychol. (2021) 12:349. doi: 10.3389 /fpsyg.2021.633533

16. Li WW, Yu H, Miller DJ, Yang F, Rouen C. Novelty seeking and mental health in Chinese university students before, during, and after the COVID19 pandemic lockdown: a longitudinal study. Front Psychol. (2020) 11:600739. doi: $10.3389 /$ fpsyg.2020.600739

17. Penner F, Ortiz JH, Sharp C. Change in youth mental health during the COVID-19 pandemic in a majority Hispanic/Latinx US sample. J Am Acad Child Adolesc Psychiatry. (2021) 60:513-23. doi: 10.1016/j.jaac.2020.12.027

18. Ciarrochi J, Scott G. The link between emotional competence and well-being: a longitudinal study. Br J Guid Counsel. (2006) 34:231-43. doi: 10.1080/03069880600583287

19. Barlow DH. Unraveling the mysteries of anxiety and its disorders from the perspective of emotion theory. Am Psychol. (2000) 55:1247-63. doi: 10.1037/0003-066X.55.11.1247

20. Martins A, Ramalho N, Morin E. A comprehensive meta-analysis of the relationship between emotional intelligence and health. Pers Individ Diff. (2010) 49:554-64. doi: 10.1016/j.paid.2010.05.029

21. Mikolajczak M, Avalosse H, Vancorenland S, Verniest R, Callens M, van Broeck $\mathrm{N}$, et al. A nationally representative study of emotional competence and health. Emotion. (2015) 15:653-67. doi: 10.1037/emo0000034

22. Mathews BL, Koehn AJ, Abtahi MM, Kerns KA. Emotional competence and anxiety in childhood and adolescence: a meta-analytic review. Clin Child Fam Psychol Rev. (2016) 19:162-84. doi: 10.1007/s10567-016-0204-3

23. Schutte NS, Malouff JM, Thorsteinsson EB, Bhullar N, Rooke SE. A metaanalytic investigation of the relationship between emotional intelligence and health. Pers Individ Diff. (2007) 42:921-33. doi: 10.1016/j.paid.2006.09.003

24. Yu L, Zhou X. Emotional competence as a mediator of the relationship between internet addiction and negative emotion in young adolescents in Hong Kong. Appl Res Qual Life. (2021) 16:1-20. doi: 10.1007/s11482-021-09912-y

25. Schutte N, Malouff J, Simunek M, McKenley J, Hollander S. Characteristic emotional intelligence and emotional well-being. Cogn Emot. (2002) 16:76985. doi: 10.1080/02699930143000482

26. Nelis D, Kotsou I, Quoidbach J, Hansenne M, Weytens F, Dupuis P, et al. Increasing emotional competence improves psychological and physical wellbeing, social relationships, and employability. Emotion. (2011) 11:354-66. doi: $10.1037 / \mathrm{a} 0021554$

27. Hofmann SG, Sawyer AT, Fang A, Asnaani A. Emotion dysregulation model of mood and anxiety disorders. Depress Anxiety. (2012) 29:409-16. doi: $10.1002 /$ da. 21888

28. Tull MT. Extending an anxiety sensitivity model of uncued panic attack frequency and symptom severity: the role of emotion dysregulation. Cogn Ther Res. (2006) 30:177-84. doi: 10.1007/s10608-006-9036-7

29. Nolen-Hoeksema S, Watkins ER. A Heuristic for developing transdiagnostic models of psychopathology: explaining multifinality and divergent trajectories. Perspect Psychol Sci. (2011) 6:589-609. doi: $10.1177 / 1745691611419672$

30. Davidson RJ, Pizzagalli DA, Nitschke JB. Representation and regulation of emotion in depression: perspectives from affective neuroscience. In: Hammen
IHGC, editor. Handbook of Depression. 2. New York, NY: Guilford Press (2009). p. 218-48.

31. Ellis A. The revised ABC's of rational-emotive therapy (RET). J Rational Emot Cogn Behav Ther. (1991) 9:139-72. doi: 10.1007/BF01061227

32. Li HC, Chung OK, Ho KY. Center for epidemiologic studies depression scale for children: psychometric testing of the Chinese version. J Adv Nurs. (2010) 66:2582-91. doi: 10.1111/j.1365-2648.2010.05440.x

33. Jiang L, Wang Y, Zhang Y, Li R, Wu H, Li C, et al. The reliability and validity of the Center for Epidemiologic Studies Depression Scale (CES-D) for Chinese university students. Front Psychiatry. (2019) 10:315. doi: 10.3389/fpsyt.2019.00315

34. Su L, Wang K, Fan F, Su Y, Gao X. Reliability and validity of the screen for child anxiety related emotional disorders (SCARED) in Chinese children. J Anxiety Disord. (2008) 22:612-21. doi: 10.1016/j.janxdis.2007.05.011

35. Wang K, Su LY, Zhu Y, Zhai J, Yang ZW, Zhang JS. Norms of the screen for child anxiety related emotional disorders in Chinese urban children. Chin J Clin Psychol. (2002) 10:270-2.

36. Shek DT, Siu AM, Lee TY. The Chinese positive youth development scale: a validation study. Res Soc Work Pract. (2007) 17:380-91. doi: $10.1177 / 1049731506296196$

37. Shek DT, Ma CM. Dimensionality of the Chinese positive youth development scale: confirmatory factor analyses. Soc Indicat Res. (2010) 98:41-59. doi: 10.1007/s11205-009-9515-9

38. Luo W, Zhong BL, Chiu HFK. Prevalence of depressive symptoms among Chinese university students amid the COVID-19 pandemic: a systematic review and meta-analysis. Epidemiol Psychiatr Sci. (2021) 30:e31. doi: 10.1017/S2045796021000202

39. Yin T, Li Y, Ying Y, Luo Z. Prevalence of comorbidity in Chinese patients with COVID-19: Systematic review and meta-analysis of risk factors. BMC Infect Dis. (2021) 21:1-13. doi: 10.1186/s12879-021-05915-0

40. Corp. I. IBM SPSS. Statistics for Windows. Armonk, NY: IBM Corp (2017).

41. Arbuckle JL. Amos (Version 23.0) [Computer Program]. Chicago, IL: IBM SPSS (2014).

42. Hou KT, Wen ZL, Cheng ZJ. Structural Equation Model and Its Application. Beijing: Educational Science Publishing House (2002).

43. Hu LT, Bentler PM. Fit indices in covariance structure modeling: sensitivity to underparameterized model misspecification. Psychol Methods. (1998) 3:42453. doi: 10.1037/1082-989X.3.4.424

44. Chen G, Gong J, Qi Z, Zhong S, Su T, Wang J, et al. The psychological status of general population in Hubei province during the COVID-19 outbreak: a cross-sectional survey study. Front Public Health. (2021) 9:203. doi: $10.3389 /$ fpubh.2021.622762

45. Jian X, Liu C. Infodemic versus pandemic factors associated to public anxiety in the early stage of the COVID-19 outbreak: a cross-sectional study in China. Front Public Health. (2021) 9:723648. doi: 10.3389/fpubh.2021.723648

46. Ciarrochi JV, Deane FP. Emotional competence and willingness to seek help from professional and nonprofessional sources. Br J Guid Counsel. (2001) 29, 233-246. doi: 10.1080/03069880124843

47. Yildirim M, Güler A. COVID-19 severity, self-efficacy, knowledge, preventive behaviors, and mental health in Turkey. Death Stud. (2020) 44:1-8. doi: 10.1080/07481187.2020.1793434

48. Abdi R, Pak R. The mediating role of emotion dysregulation as a transdiagnostic factor in the relationship between pathological personality dimensions and emotional disorders symptoms severity. Pers Individ Diff. (2019) 142:282-7. doi: 10.1016/j.paid.2018.09.026

49. Paulus DJ, Gallagher MW, Bartlett BA, Tran J, Vujanovic AA. The unique and interactive effects of anxiety sensitivity and emotion dysregulation in relation to posttraumatic stress, depressive, and anxiety symptoms among trauma-exposed firefighters. Comprehens Psychiatry. (2018) 84:54-61. doi: 10.1016/j.comppsych.2018.03.012

50. Yuan G, Liu Z. Longitudinal cross-lagged analyses between cyberbullying perpetration, mindfulness and depression among Chinese high school students. J Health Psychol. (2019) 26:1872-81. doi: 10.1177/1359105319890395

51. Shi W, Shen Z, Wang S, Hall BJ. Barriers to professional mental health helpseeking among Chinese adults: a systematic review. Front Psychiatry. (2020) 11:442. doi: 10.3389/fpsyt.2020.00442 
52. Frias CE, Cuzco C, Martín CF, Pérez-Ortega S, Triviño López JA, Lombraña M. Resilience and emotional support in health care professionals during the COVID-19 pandemic. J Psychosoc Nurs Ment Health Serv. (2020) 58:5-6. doi: 10.3928/02793695-20200506-02

53. Qi M, Zhou SJ, Guo ZC, Zhang LG, Min HJ, Li XM, et al. The effect of social support on mental health in Chinese adolescents during the outbreak of COVID-19. J Adolesc Health. (2020) 67:514-8. doi: 10.1016/j.jadohealth.2020.07.001

54. Lazaratou H, Dikeos DG, Anagnostopoulos DC, Soldatos CR. Depressive symptomatology in high school students: the role of age, gender and academic pressure. Commun Ment Health J. (2010) 46:289-95. doi: 10.1007/s10597-010-9296-2

55. Li J, Li J, Jia R, Wang Y, Qian S, Xu Y. Mental health problems and associated school interpersonal relationships among adolescents in China: a cross-sectional study. Child Adolesc Psychiatry Ment Health. (2020) 14:12. doi: 10.1186/s13034-020-00318-6

56. Yu H, Li M, Li Z, Xiang W, Yuan Y, Liu Y, et al. Coping style, social support and psychological distress in the general Chinese population in the early stages of the COVID-19 epidemic. BMC Psychiatry. (2020) 20:426. doi: 10.1186/s12888-020-02826-3

57. Beaupré MG, Hess U. Cross-cultural emotion recognition among Canadian ethnic groups. J Cross Cult Psychol. (2005) 36:355-70. doi: $10.1177 / 0022022104273656$

58. Watson H, Harrop D, Walton E, Young A, Soltani H. A systematic review of ethnic minority women's experiences of perinatal mental health conditions and services in Europe. PLoS ONE. (2019) 14:e0210587. doi: 10.1371/journal.pone.0210587

59. Howell A, Ding S, Gustafsson BA. Ethnicity and Inequality in China. 1st ed. London, UK: Taylor \& Francis (2020).

60. Weston R, Gore PA Jr. A brief guide to structural equation modeling. Couns Psychol. (2006) 34:719-51. doi: 10.1177/0011000006286345

61. Newman JC, Des Jarlais DC, Turner CF, Gribble J, Cooley P, Paone D. The differential effects of face-to-face and computer interview modes. Am J Public Health. (2002) 92:294-7. doi: 10.2105/AJPH.92.2.294

62. Shi W, Hall BJ. Help-seeking preferences among Chinese college students exposed to a natural disaster: a person-centered approach. Eur J Psychotraumatol. (2020) 11:1761621. doi: 10.1080/20008198.2020.17 61621

63. Hall BJ, Xiong YX, Yip PS, Lao CK, Shi W, Sou EK, et al. The association between disaster exposure and media use on post-traumatic stress disorder following Typhoon Hato in Macao, China. Eur J Psychotraumatol. (2019)10:1558709. doi: 10.1080/20008198.2018.15 58709

64. Yuan G, Xu W, Liu Z, An Y. Resilience, posttraumatic stress symptoms, and posttraumatic growth in Chinese adolescents after a tornado: the role of mediation through perceived social support. J Nerv Mental Dis. (2018) 206:130-5. doi: 10.1097/NMD.0000000000000778

65. Ciarrochi J, Scott G, Deane FP, Heaven PC. Relations between social and emotional competence and mental health: a construct validation study. Pers Individ Diff. (2003) 35:1947-63. doi: 10.1016/S0191-8869(03)00043-6
66. Kumschick IR, Beck L, Eid M, Witte G, Klann-Delius G, Heuser I, et al. READING and FEELING: the effects of a literature-based intervention designed to increase emotional competence in second and third graders. Front Psychol. (2014) 5:1448. doi: 10.3389/fpsyg.2014.01448

67. Nielsen L, Meilstrup C, Nelausen MK, Koushede V, Holstein BE. Promotion of social and emotional competence: experiences from a mental health intervention applying a whole school approach. Health Educ. (2015) 115:34956. doi: 10.1108/HE-03-2014-0039

68. Cheng P, Xia G, Pang P, Wu B, Jiang W, Li YT, et al. COVID-19 epidemic peer support and crisis intervention via social media. Commun Ment Health J. (2020) 56:786-92. doi: 10.1007/s10597-020-00624-5

69. Teo T, Shi W, Huang F, Hoi CKW. Intergenerational differences in the intention to use psychological cybercounseling: a Chinese case study. Pat Educ Counsel. (2020) 103:1615-22. doi: 10.1016/j.pec.2020.02.035

70. Torous J, Myrick KJ, Rauseo-Ricupero N, Firth J. Digital mental health and COVID-19: using technology today to accelerate the curve on access and quality tomorrow. JMIR Mental Health. (2020) 7:e18848. doi: 10.2196/1 8848

71. Yu B, Luo M, Liu M, Zhou J, Yang S, Jia P. Social capital changes after COVID-19 lockdown among youths in China: COVID-19 Impact on Lifestyle Change Survey (COINLICS). Front Public Health. (2021) 9:697068. doi: 10.3389/fpubh.2021.697068

72. Luo M, Zhang D, Shen P, Yin Y, Yang S, Jia P. COVID-19 lockdown and social capital changes among youths in China. Int J Health Policy Manag. (2021) 11:1-6. doi: 10.34172/IJHPM.2021.17

73. Jia P. A changed research landscape of youth's obesogenic behaviours and environments in the postACCOVID-19 era. Obes Rev. (2021) 22:e13162. doi: $10.1111 /$ obr. 13162

74. Zhang N, Yang S, Jia P. Cultivating resilience during the COVID-19 pandemic: a socioecological perspective. Annu Rev Psychol. (2022) 73:031857. doi: 10.1146/annurev-psych-030221-031857

Conflict of Interest: The authors declare that the research was conducted in the absence of any commercial or financial relationships that could be construed as a potential conflict of interest.

Publisher's Note: All claims expressed in this article are solely those of the authors and do not necessarily represent those of their affiliated organizations, or those of the publisher, the editors and the reviewers. Any product that may be evaluated in this article, or claim that may be made by its manufacturer, is not guaranteed or endorsed by the publisher.

Copyright (c) 2021 Shi, Yuan, Hall, Liu, Su, Zhao and Jia. This is an open-access article distributed under the terms of the Creative Commons Attribution License (CC $B Y)$. The use, distribution or reproduction in other forums is permitted, provided the original author(s) and the copyright owner(s) are credited and that the original publication in this journal is cited, in accordance with accepted academic practice. No use, distribution or reproduction is permitted which does not comply with these terms. 